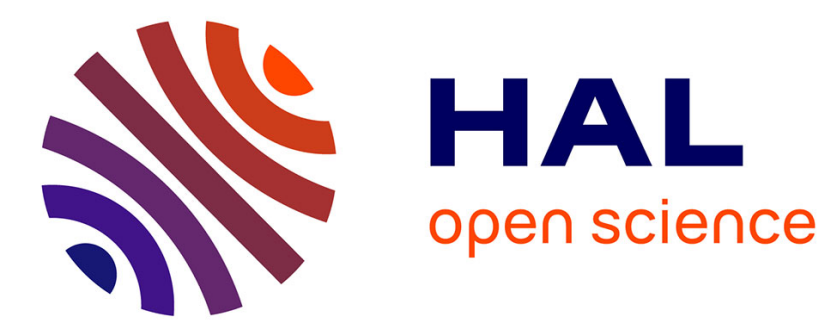

\title{
Frame based gaussian beam bouncing
}

Ihssan Ghannoum, Christine Letrou, Gilles Beauquet

\section{To cite this version:}

Ihssan Ghannoum, Christine Letrou, Gilles Beauquet. Frame based gaussian beam bouncing. EMTS 2010 : 20th URSI International Symposium on Electromagnetic Theory, Aug 2010, Berlin, Germany. pp.68-71, 10.1109/URSI-EMTS.2010.5637284 . hal-01308276

\section{HAL Id: hal-01308276 https://hal.science/hal-01308276}

Submitted on 27 Apr 2016

HAL is a multi-disciplinary open access archive for the deposit and dissemination of scientific research documents, whether they are published or not. The documents may come from teaching and research institutions in France or abroad, or from public or private research centers.
L'archive ouverte pluridisciplinaire HAL, est destinée au dépôt et à la diffusion de documents scientifiques de niveau recherche, publiés ou non, émanant des établissements d'enseignement et de recherche français ou étrangers, des laboratoires publics ou privés. 


\title{
Frame Based Gaussian Beam Bouncing
}

\author{
Ihssan Ghannoum ${ }^{\# 1}$, Christine Letrou ${ }^{* 2}$, Gilles Beauquet ${ }^{* 3}$ \\ \# Lab. SAMOVAR (UMR CNRS 5157), TELECOM SudParis \\ 9 rue Charles Fourier, 91011 Evry Cedex, France \\ 1 ihssan.ghannoumeit-sudparis.eu \\ ${ }^{2}$ christine.letroulit-sudparis.eu \\ * Surface Radar, THALES Air Systems S.A. \\ Hameau de Roussigny, 91470 Limours, France \\ ${ }^{3}$ gilles.beauquet ethalesgroup.com
}

\begin{abstract}
A general formulation based on frame re-expansions of Gaussian beam fields in the course of Gaussian beam shooting algorithms will be outlined, and closed form expressions used for frame decomposition of incident beam fields and frame change will be given. The algorithm will be tested on a specific 3d problem chosen with a view toward ground-based Radar application in semi-urban environments. The range of validity of closed form expressions for frame re-expansion coefficients will be discussed, and the accuracy of Gaussian beam summations after such re-expansions will be compared to reference solutions in cases involving diffraction.
\end{abstract}

\section{INTRODUCTION}

Paraxial Gaussian beams (GB) have long been recognized as convenient propagators for fast simulations in non uniform environments, due to their phase-space localization and caustics free behavior [1]. Their efficiency in discretizing propagating fields with an optimally low number of propagators has been demonstrated in free space contexts. Frame theory has brought additional rigor and flexibility to such discretizations [2], allowing for ultra-wideband Gaussian Beam Shooting (GBS)[3] and time-domain generalization to pulsed-beam shooting [4].

Nonetheless, efficient GBS algorithms addressing the problem of accurate, if not exact, computation of diffracted fields have not yet been developed. A recently proposed beamto-beam algorithm for half plane diffraction [5] as well as complex source point discretizations [6] are good candidates to complement the basic GBS algorithm. In this communication, we propose an alternative approach entirely based on frame discretization. This choice imposes strong constraints on beam propagators. Yet, its fully rigorous discretization scheme can be of great use either to evaluate other discretization methods or to address very general problems through efficient hybridization of GBS with full wave methods.

A general formulation based on frame re-expansions of GB fields in the course of GBS algorithms will be outlined, and closed form expressions used for frame decomposition of incident beam fields and frame change will be given. The algorithm will be tested on a specific $3 \mathrm{~d}$ problem chosen with a view toward ground-based Radar application in semi-urban environments. The range of validity of closed form expressions for frame re-expansion coefficients will be discussed, and the accuracy of GB summations after such re-expansions will be compared to reference solutions in cases involving diffraction.

\section{GBS WITH RE-EXPANSION FORMULATION}

\section{A. Outline of the algorithm}

Considering a directive antenna, its field is first decomposed on a frame of spatially wide windows. Paraxial GB are "shooted" from each window in the form of "complex rays" [3]. Within the considered environment, each beam impinging on a given surface of limited extension is then either paraxially transformed, if its field is negligible along the surface edges [7], or transformed according to the following "re-expansion" algorithm. This algorithm is performed in three successive steps:

1) decomposition of the incident field on a narrowwaisted window frame: only the windows centered on the surface of interest are considered. "Diffraction" is thus accounted for, in an approximate way similar to Physical Optics.

2) frame change: the frame coefficients for the decomposition of the incident fields on the frame of spatially wide windows are deduced from the coefficients calculated in the first step,

3) paraxial transformation: each spatially wide window represents incident fields which are paraxially reflected or refracted according to the usual geometrical optics laws generalized to complex rays.

Only two different frames are used to decompose fields on all surfaces of re-expansion:

- the first one with spatially wide windows radiating collimated beams to take advantage of the paraxial properties of the radiated beams,

- the second one with narrow-waisted windows to account for abrupt transitions on the surfaces of re-expansion.

This latter frame is only used to process field transformations on the surface but the fields radiated by narrow-waisted windows are not computed.

\section{B. Notations}

A frame of Gaussian windows in $L_{2}(\mathbb{R})$, called "Gabor frame", is a set of Gaussian functions defined by translations in space and spectrum. The "mother" window can be taken 
as:

$$
\psi(x)=\sqrt{\frac{\sqrt{2}}{L}} e^{-\pi \frac{x^{2}}{L^{2}}}
$$

and the translated windows are denoted by:

$$
\psi_{\boldsymbol{\mu}}(x)=\psi(x-m \bar{x}) e^{i n \bar{k}_{x}(x-m \bar{x})}, \quad \boldsymbol{\mu}=(m, n) \in \mathbb{Z}^{2}
$$

where $\bar{x}$ and $\bar{k}_{x}$ are respectively the spatial and spectral domain translation steps. The set of $\psi_{\boldsymbol{\mu}}$ windows is a frame in $L_{2}(\mathbb{R})$ if and only if $\bar{x} \bar{k}_{x}=2 \pi \nu$ with $\nu<1$ (oversampling factor) [8]. For better localization properties, we use so-called balanced frames, i.e. with $\bar{x}=L \sqrt{\nu}$. The re-expansion algorithm will be presented in the $3 \mathrm{~d}$ space. The frames used to represent fields on surfaces are in $L_{2}\left(\mathbb{R}^{2}\right)$, and they are constructed as products of frames in $L_{2}(\mathbb{R})$. The $\psi_{\boldsymbol{\mu}}$ frame windows in $L_{2}\left(\mathbb{R}^{2}\right)$, with $\boldsymbol{\mu}=(m, n, p, q) \in \mathbb{Z}^{4}$ and $\boldsymbol{x}=(x, y) \in \mathbb{R}^{2}$ are defined as:

$$
\Psi_{\boldsymbol{\mu}}(\boldsymbol{x})=\psi_{x \mid m, n}(x) \psi_{y \mid p, q}(y)
$$

$\left\{\psi_{x \mid m, n}\right\}$ and $\left\{\psi_{y \mid p, q}\right\}$ are frames in $L_{2}(\mathbb{R})$. In the following, all the frame parameters will be referred to frames in $L_{2}(\mathbb{R})$, and indexed by $x$ and $y$.

With reference to the re-expansion algorithm outlined in II-A, we shall denote $\left\{\Psi_{\boldsymbol{\mu}}, \boldsymbol{\mu}=(m, n, p, q) \in \mathbb{Z}^{4}\right\}$ the frame of spatially wide windows and $\left\{\Psi_{\boldsymbol{\mu}^{\prime}}^{\prime}, \boldsymbol{\mu}^{\prime}=\left(m^{\prime}, n^{\prime}, p^{\prime}, q^{\prime}\right) \in\right.$ $\left.\mathbb{Z}^{4}\right\}$ the frame of narrow-waisted windows. Also, $a_{\boldsymbol{\mu}^{\prime}}^{\prime}$ (resp. $a_{\mu}$ ) will denote the frame coefficients of field decomposition on the frame of narrow-waisted (resp. spatially wide) windows. All the frame parameters will be primed when referred to the frame of narrow-waisted windows $\left(\nu_{x}^{\prime}, \bar{x}^{\prime}, \bar{k}_{x}^{\prime}, \nu_{y}^{\prime}, \bar{y}^{\prime}, \bar{k}_{y}^{\prime} \ldots\right)$.

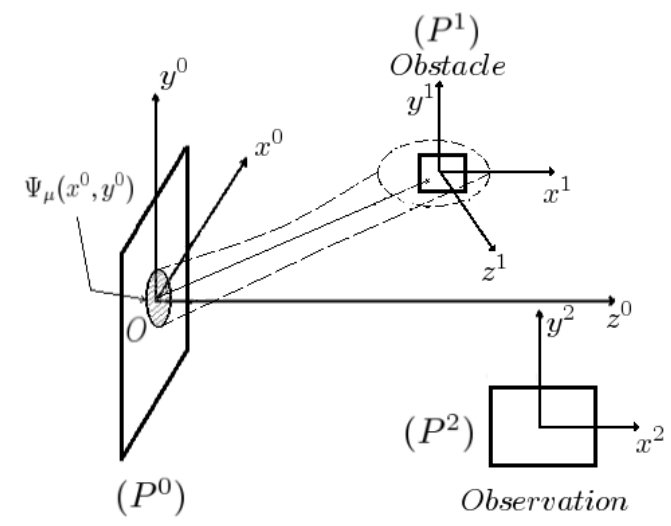

Fig. 1. Scenario used to formulate the re-expansion algorithm for one bounce

We present the re-expansion algorithm in the case of one bounce, with an incident beam impinging on a planar obstacle, as shown in Fig. 1. The incident beam is radiated into the $z^{0}>$ 0 half space by a spatially wide frame window defined in the $P^{0}\left(O, x^{0}, y^{0}\right)$ plane. The plane of de-composition is denoted $P^{1}$ and we define an associated coordinate system such that $P^{1}=\left(O^{1}, x^{1}, y^{1}\right)$. A limited size obstacle occupies a finite area in this plane. The spectral variables related to the space variables $\boldsymbol{x}^{i}=\left(x^{i}, y^{i}\right)(i=0,1)$ are noted $\boldsymbol{k}_{\boldsymbol{x}^{i}}=\left(k_{x^{i}}, k_{y^{i}}\right)$ and the $z^{i}$ component of a wavevector $\boldsymbol{k}$ is denoted by $k_{z^{i}}$. An observation plane $P^{2}$ is defined, where the fields will be computed after interacting with the obstacle.

\section{Decomposition of the incident field on the narrow-waisted} window frame

Frame coefficients can be obtained through various algorithms, among which projection on dual frame windows yields the representation with minimum energy, hence the best localization of the fields radiated from the frame expansion. In the following, we use this projection algorithm, which is greatly simplified by approximating dual frame functions by Gaussian windows [3].

The $a_{\boldsymbol{\mu}^{\prime}}^{\prime}$ coefficients are obtained from a projection integral in the spectral domain. The spectrum of the incident beam field in the plane $P^{1}$ is given by:

$$
\widetilde{\Psi}_{\boldsymbol{\mu}}^{1}\left(\boldsymbol{k}_{\boldsymbol{x}^{1}}\right)=\widetilde{\Psi}_{\boldsymbol{\mu}}\left(\boldsymbol{k}_{\boldsymbol{x}^{0}}\right) \frac{k_{z^{0}}}{k_{z^{1}}} e^{i \boldsymbol{k} \cdot \overrightarrow{O^{0} O^{1}}}
$$

The projection integral of this spectrum on the approximate dual frame functions of the transforms of spatially narrow windows, $\frac{\nu_{x}^{\prime} \nu_{y}^{\prime}}{\left\|\widetilde{\Psi}^{\prime}\right\|^{2}} \widetilde{\Psi}_{\boldsymbol{\mu}^{\prime}}^{\prime}\left(\boldsymbol{k}_{\boldsymbol{x}^{1}}\right)$, writes as:

$$
a_{\boldsymbol{\mu}^{\prime}}^{\prime}=\int_{-\infty}^{\infty} \widetilde{\Psi}_{\boldsymbol{\mu}}^{1}\left(\boldsymbol{k}_{\boldsymbol{x}^{1}}\right) \frac{\nu_{x}^{\prime} \nu_{y}^{\prime}}{\left\|\widetilde{\Psi}^{\prime}\right\|^{2}} \widetilde{\Psi}_{\boldsymbol{\mu}^{\prime}}^{\prime \times}\left(\boldsymbol{k}_{\boldsymbol{x}^{1}}\right) d \boldsymbol{k}_{\boldsymbol{x}^{1}}
$$

Using a paraxial approximation to perform this integral yields:

$$
a_{\boldsymbol{\mu}^{\prime}}^{\prime}=2 \pi c_{0} f_{\boldsymbol{\mu}^{\prime}}\left(\boldsymbol{k}_{\boldsymbol{x}^{1}}\right) e^{i g_{n q}} \frac{\exp \left(-\frac{i}{2} \boldsymbol{g}_{n q}^{\prime T} \boldsymbol{G}_{n q}^{\prime \prime}-1 \boldsymbol{g}_{n q}^{\prime}\right)}{\sqrt{-\operatorname{det} \boldsymbol{G}_{n q}^{\prime \prime}}}
$$

with:

$$
\begin{aligned}
c_{0} & =4 \pi \sqrt{\sigma_{x} \sigma_{x}^{\prime} \sigma_{y} \sigma_{y}^{\prime}} \frac{\nu_{x}^{\prime} \nu_{y}^{\prime}}{\left|\psi_{x}^{\prime}\right|^{2}\left|\psi_{y}^{\prime}\right|^{2}} \\
f_{\boldsymbol{\mu}^{\prime}}\left(\boldsymbol{k}_{\boldsymbol{x}^{1}}\right) & \left.=\frac{k_{z^{0}}}{k_{z^{1}}} \exp \left[-\frac{\sigma_{x}^{\prime 2}}{2}\left(k_{x^{1}}-n^{\prime} \bar{k}_{x}^{\prime}\right)^{2}-\frac{\sigma_{y}^{\prime 2}}{2}\left(k_{y^{1}}-q^{\prime} \bar{k}_{y}^{\prime}\right)^{2}\right)\right] \\
g\left(\boldsymbol{k}_{\boldsymbol{x}^{1}}\right) & =0.5 i\left[\sigma_{x}^{2}\left(k_{x^{0}}-n \bar{k}_{x}\right)^{2}+\sigma_{y}^{2}\left(k_{y^{0}}-q \bar{k}_{y}\right)^{2}\right] \\
& -\left[\left(k_{x^{0}}-n \bar{k}_{x}\right) m \bar{x}+\left(k_{y^{0}}-q \bar{k}_{y}\right) p \bar{y}\right]+\boldsymbol{k} \cdot \overrightarrow{O O^{\prime}} \\
& +\left[\left(k_{x^{1}}-n^{\prime} \bar{k}_{x}^{\prime}\right) m^{\prime} \bar{x}^{\prime}-\left(k_{y^{1}}-q^{\prime} \bar{k}_{y}^{\prime}\right) p^{\prime} \bar{y}^{\prime}\right]
\end{aligned}
$$

$g_{n q}, \boldsymbol{g}_{n q}^{\prime}$ and $\boldsymbol{G}_{n q}^{\prime \prime}$ are the values of the function $g$ defined above, of the vector of its first partial derivatives and of the matrix of its second partial derivatives, respectively, for the $\boldsymbol{k}_{\boldsymbol{x}^{1}}$ spectral variable corresponding to $\boldsymbol{k}_{\boldsymbol{x}^{0}}=\left(n \bar{k}_{x}, q \bar{k}_{y}\right)$, the spectral translation vector of the incident beam spectrum in the $P^{0}$ plane.

This paraxial approximation is justified by the slow amplitude variation of the narrow-waisted window spectrum as compared to the incident beam spectrum.

\section{Frame change}

Using the same projection algorithm and the same approximation for dual functions as in the previous subsection, the incident field can be expressed as a sum of spatially wide 
windows, with the frame coefficients:

$$
\begin{gathered}
a_{\boldsymbol{\mu}}=\iint_{-\infty}^{\infty}\left(\sum_{\boldsymbol{\mu}^{\prime}} a_{\boldsymbol{\mu}^{\prime}}^{\prime} \Psi_{\boldsymbol{\mu}^{\prime}}^{\prime}(\boldsymbol{x})\right) \Psi_{\boldsymbol{\mu}}^{\times}(\boldsymbol{x}) d \boldsymbol{x}=\sum_{\boldsymbol{\mu}^{\prime}} C_{\boldsymbol{\mu}}^{\boldsymbol{\mu}^{\prime}} a_{\boldsymbol{\mu}^{\prime}} \\
\text { with } C_{\boldsymbol{\mu}}^{\boldsymbol{\mu}^{\prime}}=\frac{\nu_{x} \nu_{y}}{\|\Psi\|^{2}} \iint_{-\infty}^{\infty} \Psi_{\boldsymbol{\mu}^{\prime}}^{\prime}(\boldsymbol{x}) \Psi_{\boldsymbol{\mu}}^{\times}(\boldsymbol{x}) d \boldsymbol{x}
\end{gathered}
$$

The matrix $\boldsymbol{C}$, with elements $C_{\boldsymbol{\mu}}^{\boldsymbol{\mu}^{\prime}}$ can be viewed as a "frame change" matrix.

Following (3) the integral expression of $C_{\boldsymbol{\mu}}^{\boldsymbol{\mu}^{\prime}}$ in (7) is the product of two integrals on $\mathbb{R}$ of the form:

$$
C_{m, n}^{m^{\prime}, n^{\prime}}=\int_{-\infty}^{\infty} \psi_{m^{\prime}, n^{\prime}}^{\prime}(x) \frac{\nu}{|\psi|^{2}} \psi_{m, n}^{\times}(x) d x
$$

The integral is evaluated analytically, yielding the following closed form expression of $C_{m, n}^{m^{\prime}, n^{\prime}}$ :

$$
\begin{aligned}
& C_{m, n}^{m^{\prime}, n^{\prime}}=c_{1} e^{d+i h} \sqrt{\frac{4 \pi}{v}} \exp \left(-\frac{t^{2}}{v}\right) \\
& \text { with } c_{1}=\frac{\nu}{\sqrt{\pi \sigma^{\prime} \sigma}} ; v=\frac{1}{\sigma^{\prime 2}}+\frac{1}{\sigma^{2}} ; t=n^{\prime} \bar{k}_{x}^{\prime}-n \bar{k}_{x} \\
& d=-\frac{1}{v}\left(\frac{m^{\prime} \bar{x}^{\prime}-m \bar{x}}{\sigma^{\prime} \sigma}\right)^{2} \\
& h=-\frac{2}{v}\left(m^{\prime} \bar{x}^{\prime}-m \bar{x}\right)\left(\frac{n^{\prime} \bar{k}_{x}^{\prime}}{\sigma^{2}}+\frac{n \bar{k}_{x}}{\sigma^{\prime 2}}\right)
\end{aligned}
$$

where all the frame parameters refer to the one variable frames $\left\{\psi_{m, n}\right\}$ and $\left\{\psi_{m^{\prime}, n^{\prime}}^{\prime}\right\}$ and $\sigma=L / \sqrt{2 \pi}, \sigma^{\prime}=L^{\prime} / \sqrt{2 \pi}$.

The $\left(m^{\prime} \bar{x}^{\prime}-m \bar{x}\right)$ and $\left(n^{\prime} \bar{k}_{x}^{\prime}-n \bar{k}_{x}\right)$ terms appear as squared in negative arguments of the exponential function, which results in a sparse frame change matrix.

\section{E. Field transformations}

The use of narrow-waisted windows in the first step of the re-expansion algorithm is not only desirable for paraxial approximations of spectral domain projection integrals but it also allows for easy transformations of the incident field. Problems involving abrupt transitions on a surface can also be addressed by treating differently the fields discretized by narrow waisted windows in the different subdomains of the surface. The frame discretization of incident fields on a complex surface can serve as a starting point for a full wave method [9].

In the case of smooth enough interfaces with limited extension, the frame windows used for field re-expansion after the frame change are spectrally narrow. Their fields are thus amenable to paraxial transformation, according to the ABCD and Fresnel laws classically used for paraxial beam transformations on perfectly conducting or dielectric interfaces [7].

\section{NUMERICAL TESTS}

\section{A. Test case description}

The frame parameters for the frames of spatially wide windows in $L_{2}(\mathbb{R})$ are: $\nu=0.09, L=10 \lambda$. The translation steps are then $\bar{x}=\bar{y}=3 \lambda$ and $\bar{k}_{x}=\bar{k}_{y}=0.3 k$ with $k$ the free space wavenumber. The frame parameters for the frames of narrow-waisted windows are: $\nu^{\prime}=0.09, L^{\prime}=0.075 \lambda$. The translation steps are then $\bar{x}^{\prime}=\bar{y}^{\prime}=0.0225 \lambda$ and $\bar{k}_{x}^{\prime}=\bar{k}_{y}^{\prime}=4 k$. With these parameters, the discretization with the latter frame appears as a spatial sampling. Results obtained with different parameters will be presented at the conference.

The chosen scenario is of the type shown in Fig. 1. An incident beam, linearly polarized along $y^{0}$, is shooted from the frame of spatially wide windows in the $P^{0}$ plane. The window radiating the incident beam is indexed by $\boldsymbol{\mu}=(0,25,0,0)$. The beam is thus rotated in the $x O z$ plane, with an angle $\theta_{n, q}=48.6 \mathrm{deg}$ between the beam axis and the $z^{0}$ axis in this plane. The collimating distance of the beam is $b_{0} \cos \theta_{n, q}=$ $\left(L^{2} / \lambda\right) \cos \theta_{n, q}=66.1 \lambda$.

The obstacle plane $P^{1}$ and the plane of observation $P^{2}$ are parallel to $z^{0} O^{0} y^{0}$, situated at $x^{0}=50 \lambda$ and $x^{0}=30 \lambda$ respectively. The obstacle surface is square, centered at $y^{0}=0$ and $z^{0}=41.5 \lambda$, with side length $3 \lambda$. The distance between the beam origin and the center of the obstacle is equal to $65 \lambda$, close to the beam collimating distance. The observation region in plane $P^{2}$ is square, centered at $y^{0}=0$ and $z^{0}=60 \lambda$, with side length $80 \lambda$. The origins $O^{1}$ and $O^{2}$ of the systems of coordinates associated to planes $P^{1}$ and $P^{2}$ are in the plane $y^{0}=0$ and axes are oriented as shown in Fig. 1. All the $y$ axes are parallel, and only the $y$ component of the electric field will be considered in the following, and referred to as the "field".

\section{B. Field re-expansion on the surface of the obstacle}

The incident beam axis is not impinging on the obstacle, nonetheless the beam is intercepted by the obstacle. Fig. 2 shows the magnitude of the incident beam field on the obstacle. Fig. 3 presents fields obtained by frame summation

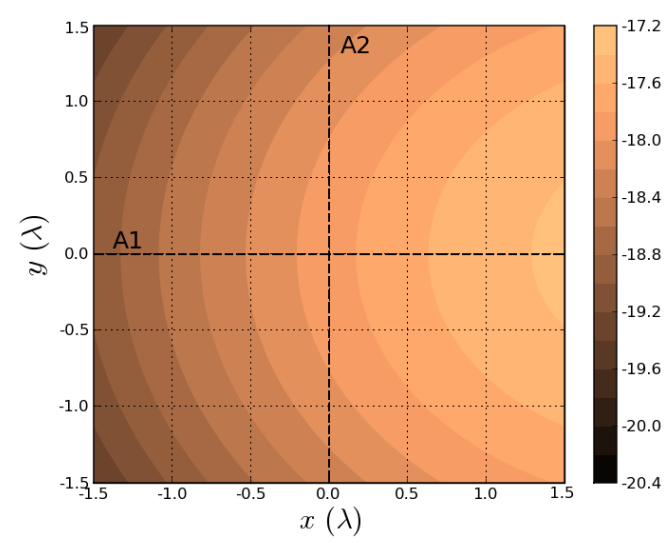

Fig. 2. Magnitude of the incident field on the obstacle

with the narrow-waisted windows and with the spatially wide frame windows, after truncation along the obstacle edges. The oscillations observed on the curve obtained with the wide windows are a consequence of spatial truncation yielding spectral widening. The number of spectrally translated wide windows required to reconstruct the non visible part of the spectral domain, which contributes to the fields in the plane of truncation, is prohibitive. As can be seen on Fig. 4 the 
visible domain $\left(-k<k_{x}<k\right)$ of truncated fields is well synthetized after re-expansion, which guarantees an accurate representation of radiated fields.

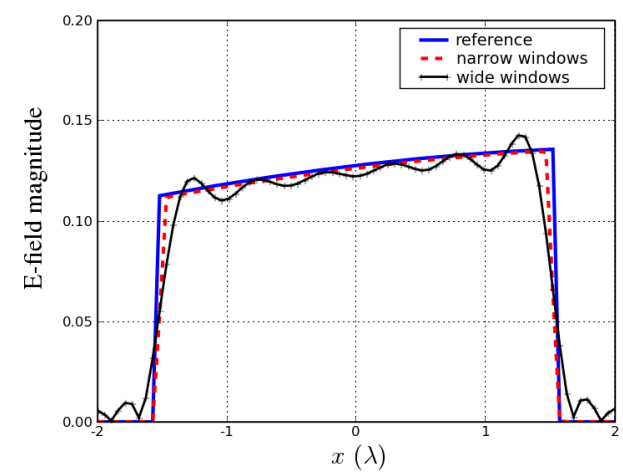

Fig. 3. Truncated field along $y^{1}=0$ in plane $P^{1}$, obtained by frame decompositions on the obstacle and by Plane Wave Spectrum (PWS) integral (reference).

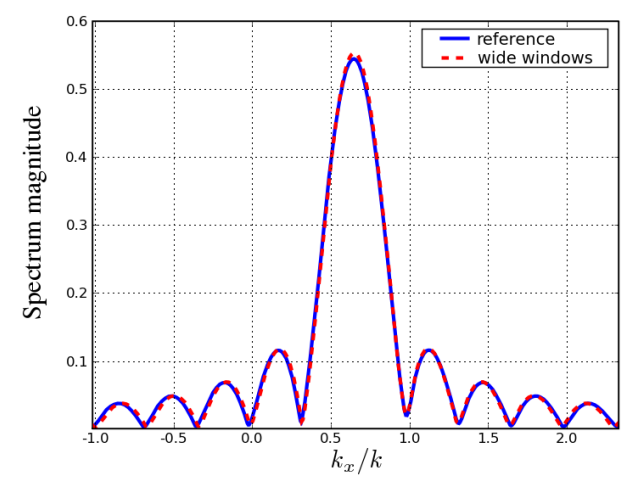

Fig. 4. Spectrum of the truncated field along $k_{y^{1}}=0$, obtained by frame reconstruction and by convolution of the incident beam spectrum with the sine cardinal accounting for truncation in plane $P^{1}$.

\section{Field propagated after the re-expansion}

The spatially wide frame windows used for field reexpansion on the obstacle radiate in the form of paraxial Gaussian beams. Diffracted fields are then obtained by summation of these beam fields in an observation region. Only beams with non negligible field in this region have to be shooted, which can be used to a priori reduce the number of frame coefficients to compute with the re-expansion algorithm. Fig. 5 presents the magnitude of the field diffracted by the obstacle, computed in plane $P^{2}$ by GBS from plane $P^{1}$. The diffraction effect is visible, and the absolute error normalized to the maximum field magnitude, obtained when comparing these results with a PWS integral reference solution is less than $-30 \mathrm{~dB}$. Reference and GBS results visibly compare very well, as illustrated in Fig. 6.

\section{CONCLUSION}

A re-expansion algorithm allowing for GB re-shooting from obstacles is presented and illustrated by the diffraction of a collimated GB on a perfectly reflecting obstacle. Encouraging results have been obtained. Other numerical results will be presented, and the choice of frame parameters will be discussed.

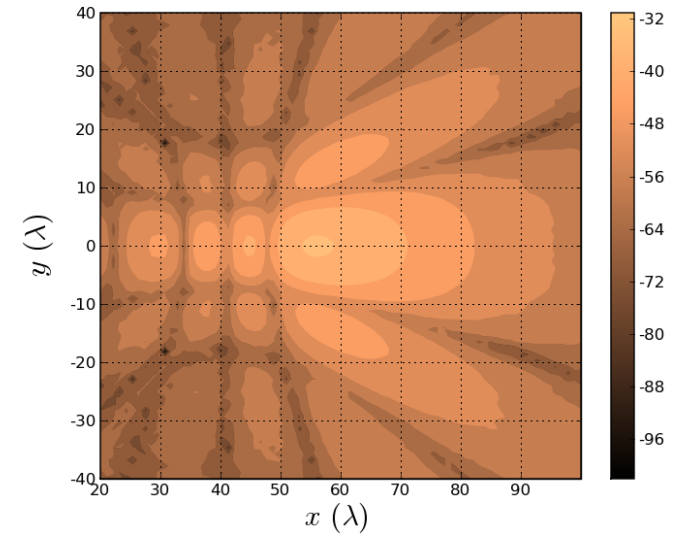

Fig. 5. Magnitude of the field computed in the plane $P^{2}$ by GBS after re-expansion (in $\mathrm{dB}$ ).

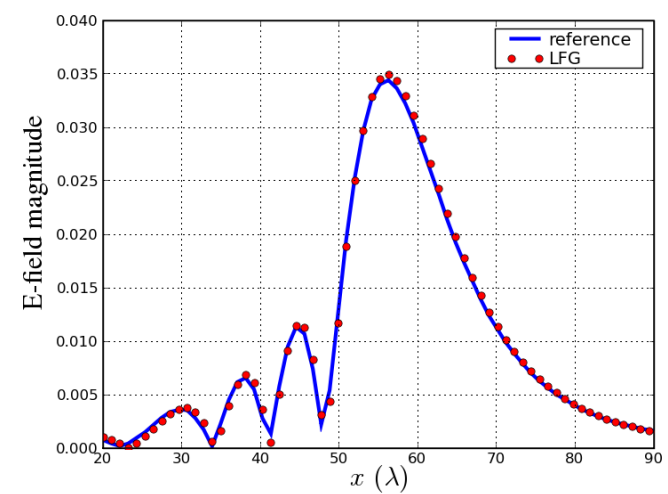

Fig. 6. Magnitude of the field computed in the plane $P^{2}$ along $y^{2}=0$ by GBS after re-expansion, compared with the reference field magnitude (PWS integral).

\section{REFERENCES}

[1] B. Steinberg, E. Heyman, and L. Felsen, "Phase-space beam summation for time-harmonic radiation from large apertures," J. Opt. Soc. Amer. A, vol. 8, pp. 41-59, Jan. 1991.

[2] D. Lugara and C. Letrou, "Alternative to Gabor's representation of plane aperture radiation," Electron. Lett., vol. 34, no. 24, pp. 2286-2287, Nov. 1998.

[3] A. Shlivinski, E. Heyman, A. Boag, and C. Letrou, "A phase-space beam summation formulation for ultrawide-band radiation," IEEE Trans. Antennas Propagat., vol. 52, no. 8, pp. 2042-2056, 2004.

[4] A. Shlivinski, E. Heyman, and A. Boag, "A pulsed beam summation formulation for short pulse radiation based on windowed radon transform (wrt) frames," IEEE Trans. Antennas Propagat., vol. 53, no. 9, pp. 30303048, 2005.

[5] M. Katsav and E. Heyman, "Gaussian beams summation representation of half plane diffraction: A full 3-d formulation," IEEE Trans. Antennas Propagat., vol. 57, no. 4, pp. 1081-1094, 2009.

[6] G. Carluccio, M. Albani, and S. Maci, "Incremental beam diffraction from flat reflectors," in EuCAP 2009 : 3rd European Conference on Antennas and Propagation, Berlin, Germany, March 2009, pp. 2119-2122.

[7] D. Lugara, A. Boag, and C. Letrou, "Gaussian beam tracking through a curved interface: comparison with a method of moments," Microwaves, Antennas and Propagation, IEE Proceedings -, vol. 150, no. 1, pp. 49-55, 2003.

[8] I. Daubechies, Ten lectures on wavelets, ser. CBMS-NSF Regional Conference Series in Applied Mathematics. Philadelphia: SIAM Press, 1992, vol. 61 .

[9] D. Lugara and C. Letrou, "Printed antennas analysis by a Gabor framebased method of moments," IEEE Trans. Antennas Propagat., vol. 50, no. 11 , pp. $1588-1597,2002$. 